\title{
NEW PUBLICATIONS
}

\section{PART I. PURE MATHEMATICS}

von Baravalle (H.). Zur Pädagogik der Physik und Mathematik. Stuttgart, Waldorf-Spielzeug und Verlag, 1928. $76 \mathrm{pp}$.

Barnett (I. A.). Analytic geometry. 2d edition. New York, Wiley, 1928. $12+345$ pp.

BATChELDER (P. M.). An introduction to linear difference equations. Published with the cooperation of the National Research Council. Cambridge, Mass., Harvard University Press, 1927. 8+209 pp.

Bessier (G.). Le calcul intégral facile et attrayant. Paris, Dunod, 1928. $8+214 \mathrm{pp}$.

Bieberbach (L.). Differential- und Integralrechnung. 3te Auflage. 2ter Band. Leipzig, Teubner, 1928. $150 \mathrm{pp}$.

BurnetT (J. C.). Hyper and ornate magic squares, 6th and 12th orders, with non-consecutive numbers. Barkston, the Author, 1928. $64 \mathrm{pp}$.

CAJORI (F.). The early mathematical sciences in North and South America. Boston, Gorham Press, 1928. 156 pp.

CAMPBEll (N. R.). An account of the principles of measurement and calculation. London, Longmans, 1928. $10+293$ pp.

Coble (A. B.). See National Research Council.

DE Comberousse (C.). Cours de mathématiques. 7e édition, entièrement refondue, par R. de Montessus de Ballore. Tome II, partie 2. Paris, Gauthier-Villars, 1928.

Doenlemann (K.). Grundzüge der Perspektive nebst Anwendungen. 3te Auflage. Leipzig, Teubner, 1928.

Donadt (A.). See LübSen (H. B.).

Drews (A.). Lehrbuch der Logik. Berlin, Stilke, 1928. 11+544 pp.

Emch (A.). See National Research Council.

GAUSS (C. F.). Anziehung eines elliptischen Ringes. (Ostwalds Klassiker der exakten Wissenschaften.) Leipzig, Akademische Verlagsgesellschaft, 1927.

GrifFIN (F. L.). Mathematical analysis. Higher course. London, Harrap, 1928. $10+512 \mathrm{pp}$.

Haussner (R.). Analytische Geometrie der Ebene. (Sammlung Göschen.) Berlin, de Gruyter, 1928. 164 pp.

JACOBI (C. G. J.). Theorie der elliptischen Funktionen. (Ostwalds Klassiker der exakten Wissenschaften.) Leipzig, Akademische Verlagsgesellschaft, 1927.

KöNIG (R.) und KRafFT (M.). Elliptische Funktionen. Berlin, de Gruyter, 1928. $263 \mathrm{pp}$.

KRAFFT (M.). See KöNig (R.).

Lefschetz (S.). See National Research Council.

Lindemann $(F$.$) . Untersuchungen über den Fermatschen Satz. München,$ Selbstverlag, 1928. 
LƯBSEN (H. B.). Ausführliches Lehrbuch der analytischen oder höheren Geometrie zum Selbstunterricht. 17te Auflage, völlig neu bearbeitet von A. Donadt. Leipzig, Brandtstetter, 1928. 7+291 pp.

Lurquin (C.). Sur la formule de Laplace. Bruxelles, 1928. 8 pp.

MAGGI (G. A.). Geometria del movimento. 3a edizione. Bologna, Zanichelli, 1927. $258+8 \mathrm{pp}$.

de Montessus de Ballore (R.). See de Comberousse (C.).

Muika (J.). Das Fermatsche Theorem. Bukarest, F. Göbl, 1927. 2 pp.

National Research Council. Bulletin No. 63: Selected topics in algebraic geometry. Report of the Committee on Rational Transformations, V. Snyder, A. B. Coble, A. Emch, S. Lefschetz, F. R. Sharpe, C. H. Sisam. Washington, National Research Council, 1928. 395 pp.

Perrovitch (M.). Leçons sur les spectres mathématiques. Paris, GauthierVillars, 1928. $90 \mathrm{pp}$.

Piaggio (H. T.). An elementary treatise on differential equations and their applications. London, Bell, 1928. 18+256+27 pp.

Reichenbach (H.). Philosophie der Raum-Zeit-Lehre. Berlin, de Gruyter, 1928.

RUTGERS (J. G.). Inleiding tot de analytische meetkunde. Tweede druk. Eerste deel: Het platte vlak. Groningen, Noordhoff, 1928.

SCHELKUNOFF (S. A.). On certain properties of the metrical and generalized metrical groups in linear spaces of $n$ dimensions. (Dissertation, Columbia.) Hamburg, Lütcke and Wulff, 1927. 8+42 pp.

Sharpe (F. R.). See National Research Council.

Sisam (C. H.). See National Research Council.

Snyder (V.). See National Research Council.

Wieleitner (H.). Mathematische Quellenbücher. Band 3: Analytische und synthetische Geometrie. Berlin, Salle, 1928. 7+89 pp.

\section{PART II. APPLIED MATHEMATICS}

Aragon (E.). Résistance des matériaux appliquée aux constructions. 2e édition, revue par P. Chambran. Tome 1. Paris, Dunod, 1928. $8+782$ pp.

Auerbach (F.). See Graetz (L.).

Baedeker (K.). See Graetz (L.).

Baker (W. E. W.). See Disney (A. N.).

Becker (R.), Plaut (H.), und Runge (I.). Anwendungen der mathematischen Statistik auf Probleme der Massenfabrikation. Berlin, Springer, 1927. $6+117 \mathrm{pp}$.

Beyer (R.). Einführung in die Kinematik. Leipzig, Jänecke, 1928.

Boll (M.) et SAlomon (C.). Introduction à la théorie des quanta. Les equations de la mécanique et de l'électronique. Paris, Doin, 1928. $20+457 \mathrm{pp}$. 
BorchaRdT (B.). Wandlungen der Atomvorstellung. Berlin, Verlag Dietz, 1928. $85 \mathrm{pp}$.

Bóuasse (H.). Hydrodynamique générale. Paris, Delagrave, 1928. $23+500 \mathrm{pp}$.

Boussinesq (J.). Cours de physique mathématique de la Faculté des Sciences. 5e édition de l'Epilogue, revue et augmentée. Paris, Gauthier-Villars, 1928. $32 \mathrm{pp}$.

Burns (G. J.). The vector theory of electricity. Los Angeles, the Author, 1928.

Chambran (P.). See Aragon (E.).

Charbonnier (P.). Essais sur l'histoire de la balistique. Paris, Imprimerie Nationale, 1928.

Chatley (H.). Studies in molecular force. London, Griffin, 1928. $11+118$ pp.

Dahns (F.). Die Ursache und die Entstehung der Ebbe und Flut. Hamburg, Hans Christians Druckerei, 1928. 32 pp.

Delaporte (R.). Des amortissements. Paris, Gauthier-Villars, 1928. $158 \mathrm{pp}$.

Demtchenko (B.). I: Sur les cavitations solitaires dans un liquide infini. II: Sur l'influence des bords sur le mouvement d'un corps solide dans un liquide. (Thèse, Paris.) Paris, Blanchard, 1928. 125 pp.

Detlefs (H.). Darstellende Geometrie. 1tes und 2tes Heft. Frankfurt a. M., Diesterweg, 1928.

Disney (A. N.), Hill (C. F.), and Baker (W. E. W.), edited by. Origin and development of the microscope. London, Royal Microscopical Society, 1928. $11+303 \mathrm{pp}$.

Dreyer (G.). Formelsammlung zur Festigkeitslehre und Elastizitätslehre. 4te neubearbeitete und erweiterte Auflage. Leipzig, Jänecke, 1928. $7+154 \mathrm{pp}$.

Forstmann (A.) und Reppisch (H.). Der Niederfrequenzverstärker. Seine Theorie und seine praktische Anwendungen zur Sprach- und Musikverstärkung. Berlin, Schmidt, 1928. 366 pp.

FRY (T. C.). Probability and its engineering uses. London, Macmillan, 1928. $14+476 \mathrm{pp}$.

Gans (R.). See Wien (W.)

Geiger (H.) und ScheEl (K.), herausgegeben von. Handbuch der Physik. Band 3: Mathematische Hilfsmittel in der Physik. Redigiert von $\mathrm{H}$. Thirring. Band 19: Herstellung und Messung des Lichts. Redigiert von H. Konen. Berlin, Springer, 1928. $14+647+18+995$ pp.

GLASER (A.). Druckdifferenzen in stehenden Schwingungen als Ursachen der Fernkräfte. Budapest, Novak, 1928. 179 pp.

Glebel (K.). Das Pendel. Halle a. S., Zentral-Verband der Deutschen Uhrmacher, 1928. $190 \mathrm{pp}$.

GraEtz (L.), herausgegeben von. Handbuch der Elektrizität und des 
Magnetismus. Bearbeitet von F. Auerbach und K. Baedeker. Band 5, Lieferung 3 (Schluss des Werkes). Leipzig, Barth, 1928. p. $12+621-896 \mathrm{pp}$.

de Gramont (A.). La télémétrie monostatique. (Mémorial des Sciences Physiques, No. 2.) Paris, Gauthier-Villars, 1928. 64 pp.

Guillet (G. L.). Kinematics of machines. New York, Wiley, 1928. $5+250 \mathrm{pp}$.

Guyot (J.). See Lemoine (J.).

Harms (F.). See Wien (W.).

Hart (I. B.). An introduction to physical science. 2d edition. London, Oxford University Press, 1928. $12+306 \mathrm{pp}$.

Harwood (P. J.). A theory of the solar system. Brighton, the Author, 1928. $3+94 \mathrm{pp}$.

Haswell (J. E.). Horology. The science of time measurement and the construction of clocks, watches and chronometers. London, Chapman and Hall, 1928. $16+267$ pp.

Hill (C. F.). See Disney (A. N.).

JAeger (W.). Elektrische Messtechnik. Theorie und Praxis der elektrischen und magnetischen Messungen. 3te umgearbeitete Auflage. Leipzig, Barth, 1928. $24+553$ pp.

JAfFE (G.). See WiEn (W.).

JANET (P.). Leçons d'électrotechnique générale. 6e édition, revue et augmentée. Tome III. Paris, Gauthier-Villars, 1928. 4+414 pp.

Jeans (J. H.). Astronomy and cosmogony. Cambridge, University Press, 1928. $420 \mathrm{pp}$.

Joffe (A. F.). The physics of crystals. Edited by L. B. Loeb. New York, McGraw-Hill, 1928. $11+198$ pp.

Konen (H.). See Geiger (H.).

Lemorne (J.) et Guyot (J.). Cours de physique. Tome 1: Optique. Paris, Vuibert, 1928.

LODGE (O.). Der Äther und die Wirklichkeit. (Ether and reality.) Eine Reihe von Vorträgen. Aus dem Englischen übersetzt von W. Rump. Braunschweig, Vieweg, 1928. $8+89$ pp.

Loeb (L. B.). See Joffé (A. F.).

LoREntZ (H. A.). Vorlesungen über theoretische Physik an der Universität Leiden. 2ter und 3ter Band. Leipzig, Akademische Verlagsgesellschaft, 1928. $6+136+4+78 \mathrm{pp}$.

Michel (E.). Raumakustisches Merkblatt. Hannover, Verlag Curt H. Vincentz, 1927. $12 \mathrm{pp}$.

Niggli (P.). See WiEn (W.).

ORTVAY (R.). Bevezetés az anyag korpuszkulális elméletébe. (Introduction to the corpuscular theory of matter.) Part 1. Budapest, Hungarian Academy of Sciences, 1927. $294 \mathrm{pp}$. 
Plaut (H.). See Becker (R.).

Pogány (B.). Az elektromágneses tér. (The electromagnetic field.) Budapest, Athenaeum Press, 1927. 695 pp.

Porter (A. W.). See Preston (T.).

Preston (T.). The theory of light. 5th edition, edited by A. W. Porter.

London, Macmillan, 1928. $24+643 \mathrm{pp}$.

Raibaud (-) Appareils et méthodes de mesure mécaniques. Paris, Colin, 1928. $215 \mathrm{pp}$.

Reppisch (H.). See Forstmann (A.).

RUCKHABER (E.). Die Relativitätstheorie widerlegt durch das Wider spruchprinzip und die natürliche Erklärung des Michelsonversuchs Das drei dimensionale Raum-Zeit-System. Leipzig, Verlag Hillmann, 1928. $3+35$ pp.

Rump (W.). See Lodge (O.).

Runge (I.). See Becker (R.).

Rupert-Jones (J. A.). Tidal research. The adaptation of Sir Isaac Newton's tidal laws to the prediction of the height of high tides. Southampton, the Author, 1928. 20 pp.

SACKUR (O.). Lehrbuch der Thermochemie und Thermodynamik. 2te Auflage, von C. von Simson. Berlin, Springer, 1928. $16+347$ pp.

Salomon (C.). See Boll (M.).

Scheel (K.). See Geiger (H.).

Scrmid (F.). Das Zodiakallicht. Sein Wesen, seine kosmische oder tel lurische Stellung. Hamburg, Henri Grand, 1928. 10+132 pp.

Semenoff (N.) und Walther (A.). Die physikalischen Grundlagen der elektrischen Festigkeitslehre. Berlin, Springer, 1928. 7+168 pp.

von Simson (C.). See Sackur (O.).

SkIRL (W.). Elektrische Messungen. Berlin, de Gruyter, 1928. 12+459 pp.

Steward (G. C.). The symmetrical optical system. (Cambridge Tracts in Mathematics and Mathematical Physics, No. 25.) Cambridge, University Press, 1928. 8+102 pp.

Stifter (A.). See Wotruba (R.).

Strangman (C.). The origin of the solar system. Victoria, B. C., the Author, 1928. 34 pp.

Strauch (F.). Repetitorien der Mechanik. Teil 1: Gleichheitslehre. Charlottenburg, Kiepert, 1928. 52 pp.

Sugot (G.). Balistique extérieure théorique. Paris, Gauthier-Villars, 1928. $93 \mathrm{pp}$.

- Balistique intérieure théorique et tables numériques. Paris. Gauthier-Villars, 1928. 130 pp.

Thirring (H.). See Geiger (H.).

Walther (A.). See Semenoff (N.).

Weiss (E. H.). La mécanique. Paris, Hachette, 1928. 197 pp. 
WeyL (H.). Gruppentheorie und Quantenmechanik. Leipzig, Hirzel, 1928. $8+288 \mathrm{pp}$.

WiEn (W., und Harms (F.). Handbuch der Experimentalphysik. Band 7, Teil 1: P. Niggli, Kristallographische und strukturtheoretische Grundbegriffe. Band 19: Dispersion und Absorption, von G. Jaffe; Medien mit veränderlichen Brechungsindex, und Lichtzerstreuung, von R. Gans. Leipzig, Akademische Verlagsgesellschaft, 1928. $12+317+8+430 \mathrm{pp}$.

WILD (R.). Studies in magnets and magnetism. London, Stockwell, 1928. $70 \mathrm{pp}$.

Wolfers (F.). Eléments de la physique des rayons X. Paris, Hermann, 1928. $400 \mathrm{pp}$.

Wolkowitsch (D.). Applications de la géométrie à la stabilité des constructions. Tome 1. Paris, Doin, 1928. $304 \mathrm{pp}$.

Wotruba (R.) und Stifter (A.). Die Transformatoren. Theorie, Aufbau und Berechnung. München, Oldenbourg, 1928. 4+203 pp.

Zehnder (L.). Die Entwicklung des Weltalls aus mechanischen Grundlagen. Tübingen, Laupp, 1928. $6+71 \mathrm{pp}$.

ZINGLER (J.). Theorie der zusammengesetzten Waagen mit Gewichtsschale, Laufgewichtswaagen, Neigungswaagen, Balkenwaagen, Brückenwaagen. Berlin, Springer, 1928. $8+203$ pp.

ZoRETti (L.). Les principes de la mécanique classique. (Mémorial des Sciences Mathématiques, No. 30.) Paris, Gauthier-Villars, 1928. 